\title{
Reduction of Noise in Wireless Devices
}

\author{
K. Kiran \\ Assistant Professor, ECE \\ Vishnu Institute of Technology
}

\author{
M. V. R. V. Prasad \\ Assistant professor, ECE \\ Vishnu Institute of Technology
}

\author{
M. Sirisha \\ Assistant professor, ECE \\ Vishnu Institute of Technology
}

\begin{abstract}
Interference is one of the burning issues in the field of Communication. The discovery of data, interpretation and gaining knowledge from the data is a crucial phase in everyone's day to day life. Wi-Fi and Bluetooth play a crucial segment for transferring data between two domains. Wi-Fi and Bluetooth use the same unlicensed Industrial, scientific and medical band. Its frequency range is $2.4 \mathrm{GHz}$. Because of the band, interference may occur due to co-located devices. Since the utility and demand of Wi-Fi and Bluetooth devices has increased, co-located devices have also increased in number. In this paper, a new technique is proposed in which the modulation techniques to improve the performance of $\mathrm{Wi}$ $\mathrm{Fi}$ and Bluetooth co-located systems are discussed. By using least square error based estimation, BER over the Wi-Fi and Bluetooth environment is decreased. SNR gives the bit error rate of Wi-Fi and Bluetooth for each modulation technique. In this paper, different modulation techniques are performed for calculating the efficiency in the presence of interference and absence of interference.
\end{abstract}

\section{Keywords}

Wireless, Noise reduction

\section{INTRODUCTION}

Today, we are living in the living in the era of big data. Bluetooth technology and Wi-Fi technology plays a crucial part in the world of communication. Bluetooth is among the latest trends in wireless technology. It uses WPAN and operates in the range of $2.4 \mathrm{GHz}-2.4835 \mathrm{GHz}$ [1]. Bluetooth spectrum is divided into 79 channels each of $1 \mathrm{MHz}$. Another wireless technology is $\mathrm{Wi}-\mathrm{Fi}$ which we normally called as IEEE 802.11. It is a

set of MAC and PHY specifications for implementing WLAN in the frequency band. The Wi-Fi spectrum is divided into 3 wide channels [2]. In this project, interference caused due to Wi-Fi signal which is transmitted at same frequency as that of Bluetooth signal is analysed. The signal is transmitted using Frequency hoping spread spectrum and direct sequence spread spectrum.
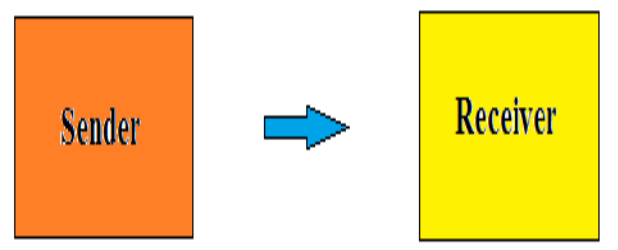

Fig.1.1. Transmission of signal from sender to receiver

\section{LITERATURE SURVEY}

There are various techniques in the world of communication to reduce the effect of information between Bluetooth and IEEE 802.11. In this paper, few of them are discussed.

\section{a. Adaptive Frequency hoping}

In this technique, Bluetooth channel adapt to the environment by identifying the interference channels. By identifying the interference channel, signal will avoid that channel. The Bluetooth device will use hop sequence for communication. When Bluetooth detects the channel which is serving the IEEE 802.11 then it will remove the channel from its hop sequence [3]. Channel identification is done by two methods. 1) Received signal strength indication (RSSI) 2) Packet error rate (PER). RSSI is more accurate when compared to PER. In this technique, the risk of collision of channels increases because the number of channels used by the Bluetooth gets reduced.

\section{b. RF isolation and Gain adjustment}

The transmit power defines the device reliability in the case of communication. When Wi-Fi device transmit higher power then more number of Bluetooth channels needs to mask in order to ensure low packet error rate (PER). Due to this, there is a need for adjusting the transmitting power of the two devices to maintain the individual links.

\section{PROPOSED SYSTEM}

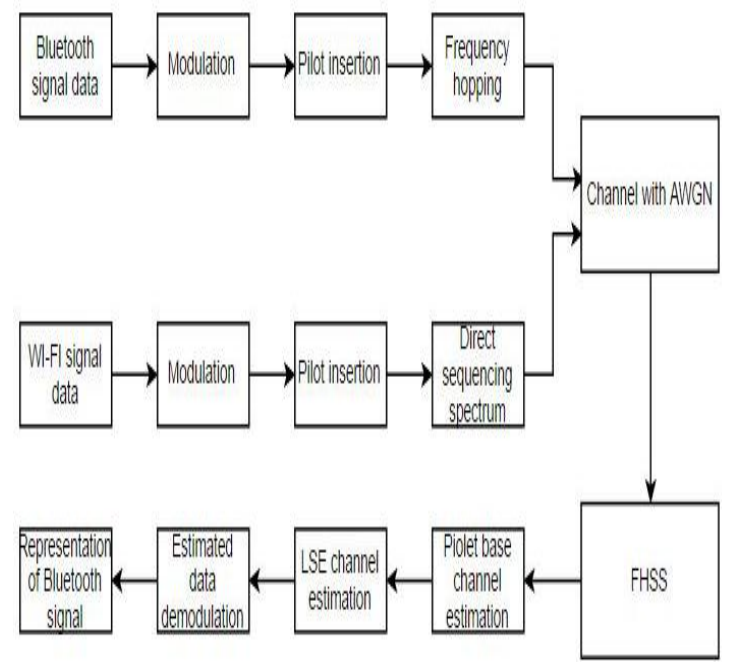

Fig.3.1. Block diagram of proposed method

\section{Bluetooth signal}

The signal is generated from the Bluetooth device which is transmitted from sender to the receiver.

\section{Modulation}

There are various types of modulation schemes. They are BPSK, QPSK, QAM etc..,

\section{BPSK Modulation}

In phase shift keying (PSK), the phase of a carrier is changed according to the modulating waveform. In BPSK, the transmitted signal is a sinusoid signal of fixed amplitude. It has fixed phase when the data is at one level and phase is shifted by 180 degree. A Binary Phase Shift Keying (BPSK) signal can be defined as

$$
V_{B}(t)=b(t) \cos 2 \pi f_{c} t
$$




\section{QPSK Modulation}

In Quadrature Phase Shift Keying (QPSK) two sinusoidal signals (sine and cosine) are taken as basic functions for modulation. Modulation is achieved by varying the phase of these sine and cosine functions depending on the message symbols [4]. In QPSK, modulation is symbol based where each symbol contains 2 bits. The QPSK modulation is given as

$$
\operatorname{Sin}(t)=2 E_{s} T-\sqrt{\cos \left(2 \pi f_{c} t+2(n-1) \pi 4\right)} \ldots(E q .3 .2)
$$

\section{QAM Modulation}

Quadrature amplitude modulation (QAM) is an analog and digital modulation scheme. It conveys two digital bit streams or two analogy message signals by modulating the amplitudes of two carrier waves using amplitude modulation (AM) or amplitude shift keying (ASK) digital modulation scheme. The two carrier waves (Quadrature Carriers), usually sinusoids, are out of phase with each other by $90^{\circ}$ [5]. The modulated waves are summed, and the resulting waveform is a combination of both amplitude-shift keying (ASK), and phase-shift keying (PSK) and in the case of digital one, combination of phase modulation (PM) and amplitude modulation (AM).

$$
\begin{array}{r}
s(t)=\sum_{m=-\infty}^{\infty} v_{c}(n) \cdot h_{t}\left(t-n T_{s}\right) \cos \left(2 \pi f_{0}\right)-v_{s}(n) . h_{t}(t \\
\left.-n T_{s}\right) \sin \left(2 \pi f_{0}\right) \ldots \ldots \ldots(E q .3 .3)
\end{array}
$$

\section{Pilot Insertion}

For the purpose of synchronization, pilot insertion is needed. Synchronization means transmission of definite signal on definite frequencies which helps the receiver to find them quickly [6].

\section{Frequency hoping}

Frequency hopping spread spectrum (FHSS) is a method of transmitting signals by rapidly switching a carrier among many frequency channels, using a pseudorandom sequence which is known to both transmitter and receiver [7].

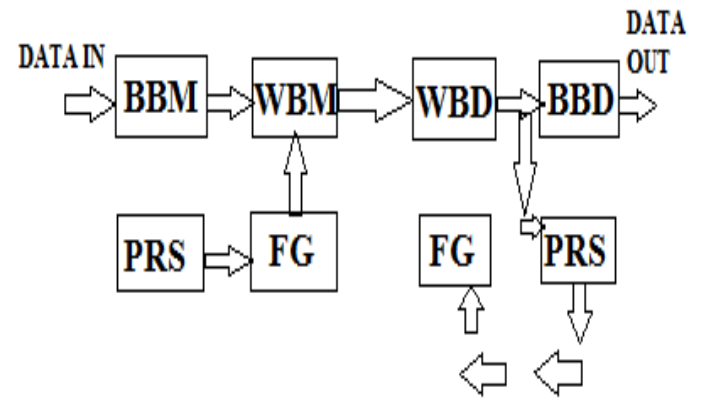

Fig.3.2.Block diagram of FHSS

Where BBM is base band modulation, WBM is wide band modulation, WBD is wide band demodulation, BBD is base band demodulation, FG is frequency generator.

\section{Direct sequence spread spectrum}

Direct Sequence Spread Spectrum is a technique in which original data signal is multiplied with a pseudo random noise spreading code. This spreading code has a higher chip rate which results in a wideband time continuous scrambled signal.

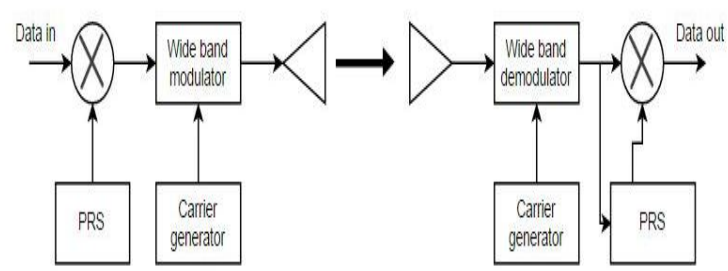

DSSS Transmitter Part

DSSS Receiver Pat

Fig.3.3.Block diagram of DSSS

\section{LSE Channel Estimation}

In Least square error estimation, only signal model is used. No probabilistic assumptions are required. It is given as [8]

$$
\mathrm{h}_{\text {lse }}=\mathrm{GR}_{1 \mathrm{se}} \mathrm{G}^{\mathrm{H}} \mathrm{U}^{\mathrm{H}} \mathrm{V}
$$

\section{Modified Channel Estimation}

The performance of LSE can be improved by considering only first L high energy channel taps. This is done by modifying the LSE. It is given as

$$
\mathrm{h}_{\text {lse }}=\mathrm{TR}_{\text {lse }}^{\prime} \mathrm{T}^{\mathrm{H}} \mathrm{U}^{\mathrm{H}} \mathrm{V}
$$

Down-Sampled Impulse Response LSE Channel Estimation

The inversion of $\mathrm{N}^{*} \mathrm{~N}$ matrix can be simplified by decreasing the sampling frequency, but ensuring the absence of aliasing. Only 2 out of 3 channel taps are used and the discarded taps are set to zero. The estimated channel in this will be

$$
\hat{h}_{d s}=\left(G^{d s, H} U^{H} U G^{d s}\right)^{-1} \cdot G^{d s, H} U^{H} V
$$

\section{RESULTS}

a. Generation and Modulation of Bluetooth signal
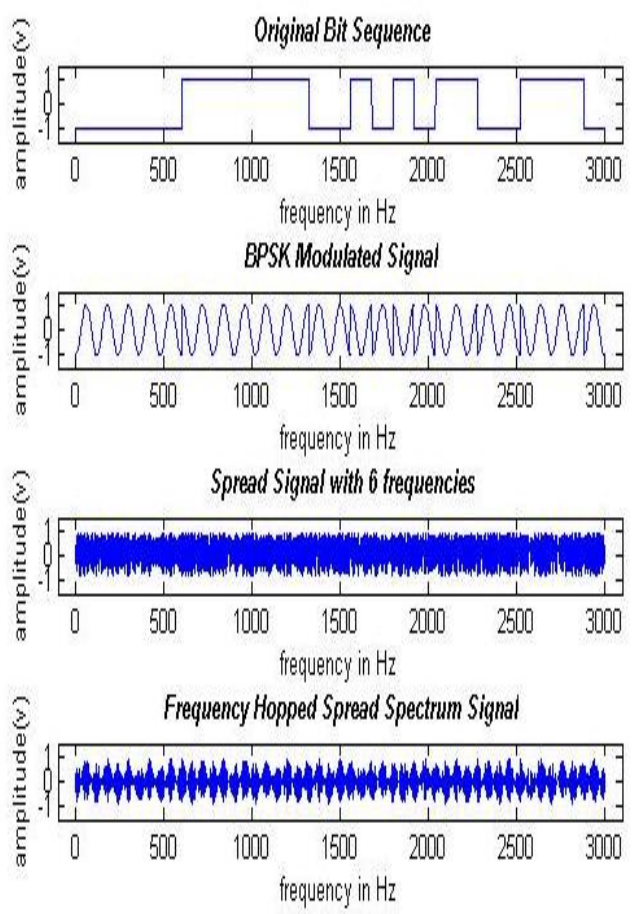

Fig.4.1. Generation and Modulation of Bluetooth signal 
b. FHSS and its FFT
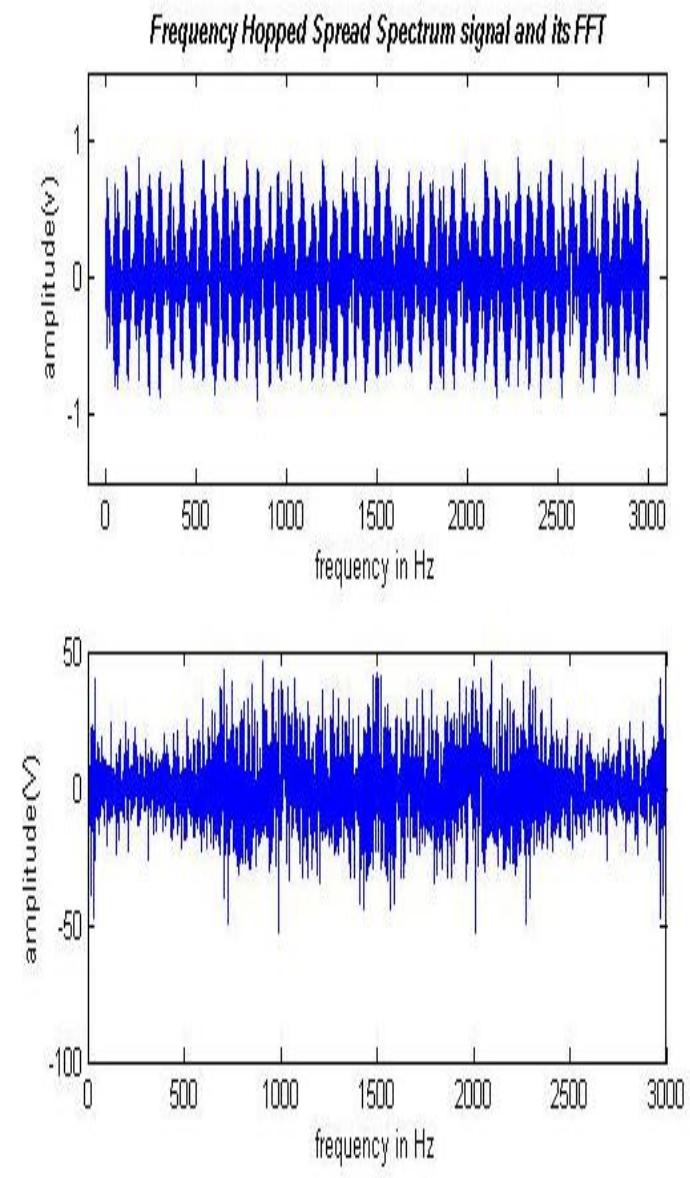

Fig.4.2. FHSS and its FFT

c. BER vs SNR Plot

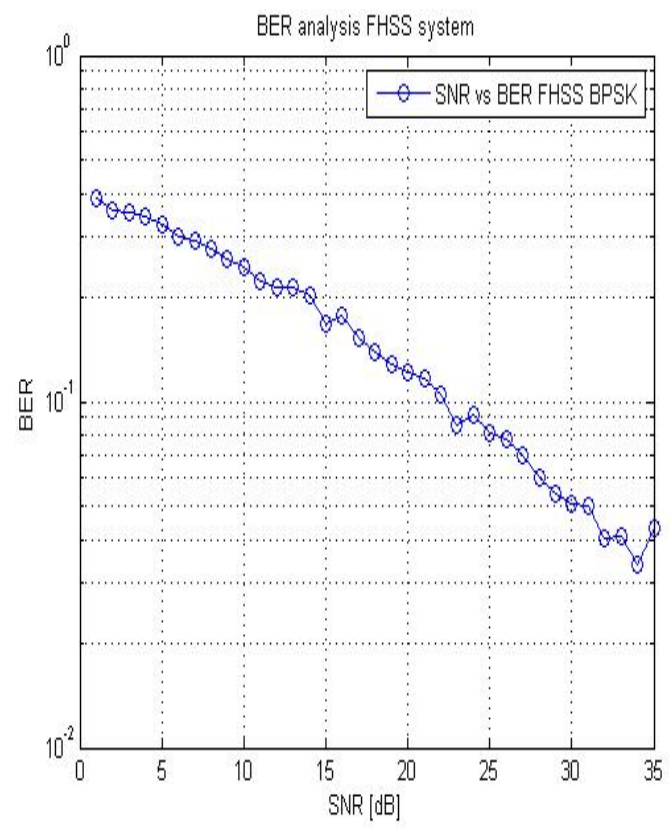

Fig.4.3. BER vs SNR Plot

\section{d. AWGN Plot}

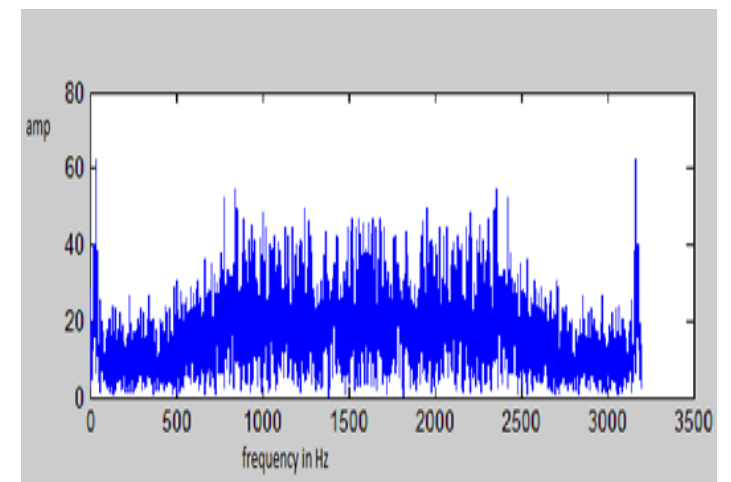

Fig.4.4. AWGN Plot

e. Performance Plot with and without interference

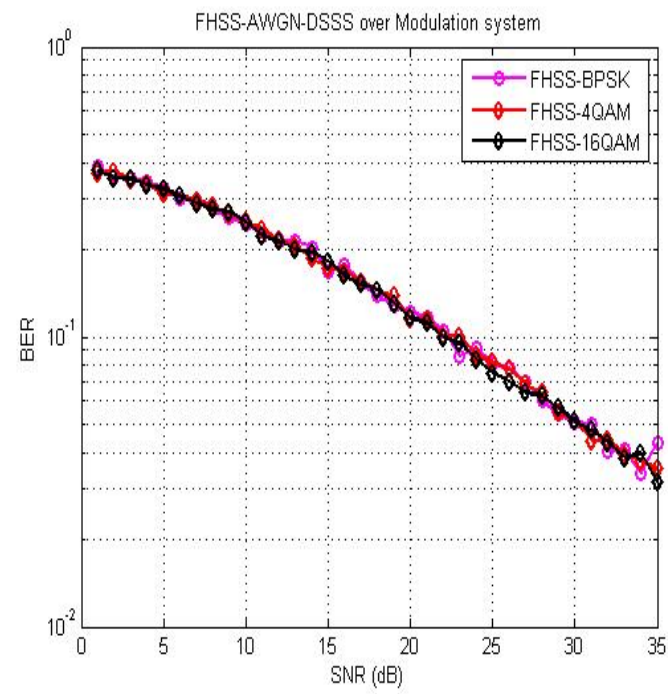

Fig.4.5. Performance Plot with interference

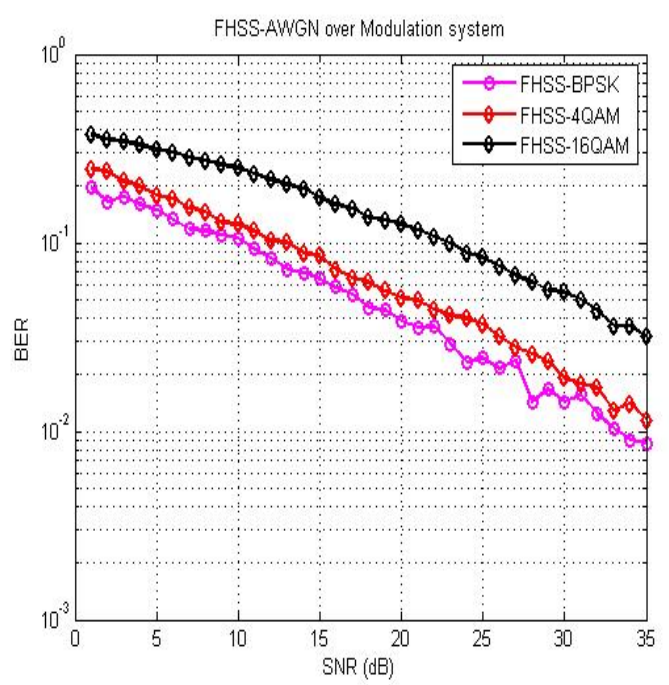

Fig.4.6. Performance Plot without interference 


\section{CONCLUSION}

In the world of communication, there is a need to avoid congestion of channels and avoid collision of transmitted signal from Bluetooth device and IEEE 802.11 or Wi-Fi networks. Use of LSE based estimation technique is a good solution for decreasing bit error rate over the wireless $2.4 \mathrm{GHz}$ Bluetooth wireless environment. By using different modulation techniques we can also improve the performance of the signal to be transmitted without interference. By using this proposed method channels for Bluetooth are provided at regular pace without any delay in the network because of its reliability.

\section{REFERENCES}

[1] Wi-Fi and Bluetooth coexistence issues and solutions for the $2.4 \mathrm{GHz}$ ISM band by Matthem B. Shoemake "Texas Instruments".

[2] Wi-Fi and Bluetooth - Interference Issues http://www.hp.com/rnd/library/pdf/WiFi_Bluetooth_coe xistance.pdf
[3] Adaptive frequency hoping by Kjetil Holstad

[4] "QPSK Modulation" Digital communications by S. Chitode

[5] Dr.Kamilo, Wireless Digital Communication Modulation and Spread Spectrum.

[6] Interference free pilot insertion for MIMO-GFDM channel estimation in wireless communication \& networking conference IEEE by Shahab Ehsanfar, Maximillian Matthe, Dan Zhang, Gerhard Fettweis.

[7] Charles Hodgdon, Adaptive Frequency Hopping for Reduced Interference between Bluetooth ${ }^{\circledR}$ and Wireless LAN. Retrieved from http://www.designreuse.com/articles/575/adaptivefrequency hopping-for reduced-interference-betweenbluetooth and-wirelesslan.html.

[8] Implementation of least square channel estimation algorithm for MIMO-OFDM system by samuli, Jari ylioinas, Markus Myllyla. 